\title{
Comparing the Social Support Which Fathers and Mothers of Preterm Infants Receive in the Neonatal Intensive Care Units
}

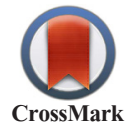

\author{
Zahra Ahmadi ${ }^{1}$, Mona Joz Mohtashami2 ${ }^{2}$, Na imeh Seyyed Fatemi ${ }^{3}$, Hamid Haghani ${ }^{4}$ \\ 1. Department of Community Health Nursing Education, School of Nursing \& Midwifery, Iran University of Medical Sciences, Tehran, Iran. \\ 2. Department of Nursing, School of Nursing and Midwifery, Shahroud University of Medical Sciences, Shahroud, Iran. \\ 3. Center for Nursing Care Research, School of Nursing \& Midwifery, Iran University of Medical Sciences, Tehran, Iran. \\ 4. Department of Biostatistics, School of Health Management and Information Sciences, Iran University of Medical Sciences, Tehran, Iran.
}

Citation: Ahmadi, Z, Joz Mohtashami, M, Seyyed Fatemi, N \& Haghani, H 2016, 'Comparing the Social Support Which Fathers and Mothers of Preterm Infants Receive in the Neonatal Intensive Care Units', Journal of Client-Centered Nursing Care, vol. 2, no. 2, pp. 83-88. https://doi. org/10.32598/jecnc. 2.2 .83

: https://doi.org/10.32598/jcenc.2.2.83

Article info:

Received: 30 Nov. 2015

Accepted: 13 Feb. 2016

Keywords:

Social support, Parents, Preterm infants

\begin{abstract}
A B S T RA C T
Background: The birth of a preterm infant is a stressful experience for parents. The nurse's support for the family of such newborns seems to be important and necessary. This study aimed to examine the social support received by the fathers as compared with the mothers of preterm infants hospitalized in the neonatal intensive care unit (NICU).

Methods: This was a comparative, descriptive study. The study population included all parents of preterm infants hospitalized in the NICUs of 3 educational treatment centers affiliated to Tehran University of Medical Sciences during the first 6 months of the year 2014. Study sample consisted of 130 couples selected through the convenient sampling method, and data collection tool was Tarkan et al. social support questionnaire. Then, the obtained data were analyzed using the Independent $t$ test by SPSS version 20.

Results: The results showed that the most social support with the mean(SD) score of $69.0(6.3)$ was received by the mothers, and 57.0(2.3) for fathers was emotional support and the least level of social support with the mean score of $80.0(3.3)$ for the mothers and $65.0(16.3)$ for fathers was tangible support, and mothers with mean score 65.0(52.3) as compared with fathers 56.0(21.3), received more support in all 3 aspects of social support, i.e. affirmative, tangible, and emotional.

Conclusion: Our findings indicate that parents are desirous of more social support, especially with regard to the tangible support. Therefore, planning is required for promotion and creation of opportunities for the nurses to provide more supports to parents at NICUs, especially to fathers who usually receive less support. It is recommended that nurses improve their effective and mutual communication and interaction with parents.
\end{abstract}

\section{* Corresponding Author:}

Mona Joz Mohtashami, MSc.

Address: Department of Nursing, School of Nursing and Midwifery, Shahroud University of Medical Sciences, Shahroud, Iran Tel: +98 (233) 2395054

E-mail: mohtashami@shmu.ac.ir 


\section{Background}

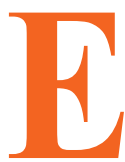

ach year, 15 million preterm infants are born throughout the world, of whom no more than one million survive (Blencowe et al. 2012). Many newborn infants experience difficult problems and need special cares. Birth of preterm infant creates a stressful and critical situation for parents (Arnold et al. 2013). They are often confronted with challenges to admit their parental role. The challenges that may have long term and lasting impacts on the function of the family.

Nurses have a unique opportunity to help parents during this difficult experience (Cleveland 2008). Parents of preterm infants, because of these challenges and stresses need social consideration and support (Ibrahimi et al. 2011). It is important to reduce parents'stress during hospitalization of their preterm infants. If sufficient social support is provided during the hospitalization of preterm infants, their parents' stress will truly decrease (Schappin et al. 2013). Support of parents is synonymous with family-centered care and aims to enable the family to assume roles and support family in stressful situations (Valizadeh et al. 2012). The task of neonatal intensive care unit (NICU) is to perform proper care under conditions that are not only suitable for the newborn but also supportive for parents (Sikorova \& Kucova 2012). In the NICU, stressful factors for the parents are the experience of preterm newborn birth, the appearance of the preterm newborn, complicated illnesses, difficult experience of the parenthood, rigid and inflexible hospital policies, challenging relations with nurses and health care providers, economic problems, transportation and commuting problems, and their privacy protection. Based on a study conducted by Johnson in 2008 , more than $36 \%$ of parents have stated that they have not found a person who can address their problems, $29 \%$ of these parents have objected to the inadequacy of protection of privacy, and $25 \%$ were unable to discuss more issues with the health staff. Since any of these factors greatly affects parents, resolution of these issues is important (Johnson et al. 2005).

Support of parents by nurses is very important for the parents to deal with stress. Based on Kahn's conceptual definition (1979), social support is interpersonal reactions which include emotional support, affirmative support, and interconnected support. Emotional support is the sense of loving, admiration, and respect. It includes caring, sympathy, love, and sincerity. Affirmative support emphasizes functions and statements. Interconnected support includes financial, informational supports, and saving time (Oommen et al. 2011).
In a neonatal ward, nurses rarely work with the aim of providing family-centered care. Most of their energy concentrate on meeting patients' needs. Usually, they focus on the technical details of clinical care, and the family is left unnoticed (Valizadeh et al. 2012). In a research conducted by Oomen et al. it was shown that during the critical period of newborns' hospitalization at NICU, social support of their parents is of utmost importance, and nurses should be encouraged to acquire communication skills and create opportunities for social support of parents (Oommen et al. 2011).

Being under great pressure, fathers need to create balance between their jobs and responsibilities in the NICU. Fathers prefer to go back sooner to their works and be less present in the NICU (Johnson 2008) because of their insufficient confidence, no knowledge about preterm newborn's care, complex technology in NICU, less emotional attachment, and being uncomfortable in the NICU environment.

Few studies have been conducted on the social support needed by parents of preterm newborns. Studies have shown that the social support for such parents is poor (Oommen et al. 2011). One of the most important steps needed to be taken by nurses is to examine ways of establishing communication with preterm newborns parents and supporting them. Nurses engaged in the intensive care units need to create opportunities for participation of parents and supporting them (Johnson 2008). Considering that every year a great number of newborn babies are hospitalized in NICUs, and this issue can cause anxiety in parents and disrupt their parental role, effective measures should be taken to develop programs and situations that would provide social support for parents and consider more their needs.

Therefore, a step, even though small, can be taken to reduce stress and anxiety in preterm infants' parents. Furthermore, parental role in father and mother is different, for example, fathers may spend less time with their infants as compared with the mothers, and in the meantime, in the intensive care unit, mothers have to care for their newborns, and spend far more time with them. Parents may have different expectations from one another, and thus, social support can have different effects on each parent. Therefore, one of the parents (father or mother) may need more support and consideration by nurses to improve parental role and reduce stress. Nurses who are aware of the opinion of each parent about the social support, can establish more effective communication with them and taking their needs into account. 
Considering the importance of family-centered care in managing the health of preterm infants and scarcity of studies in our country in this regard, the present study was conducted for the purpose of comparing the view of fathers and mothers regarding social support received by parents from nurses in NICU and utilizing the study results in future planning of the health system.

\section{Materials \& Methods}

The present study was of the comparative-descriptive type and the study population included all parents of preterm infants hospitalized at NICUs of 3 treatment-educational centers of Arash, Valiasr, and Bahrami in Tehran, Iran. In total, 260 individuals comprised the study sample. Both parents of each newborn took part in our study. The sample size was calculated as 130 and sampling was performed through the convenient sampling method. Inclusion criteria were ability of the parents to read and write Farsi to fill out the questionnaire, absence of any congenital anomalies and neural illnesses in the newborns, no previous experience of newborn hospitalization in the NICU, or the death of the newborn, (without impact on parents viewpoint), and passage of at least 3 days of hospitalization of the newborn in the NICU.

Data collection was performed by 21 -item social support questionnaire. The first 7 questions were about the affirmative support, 6 next questions were related to tangible support, and 8 questions associated with emotional support. Total scores ranged from 0 which indicated that the subject needed no support to 6 which indicated the highest level of social support needed. The validity of social support measure was determined through the content validity method. In this way, the English questionnaire was translated into Farsi and the translated version together with its English version and explanations about the study aims and study method were reviewed and examined by 10 members of the scientific faculty who were experts on the issue. Then, their recommended modifications were implemented under the supervision of student advisers. To evaluate the reliability of the questionnaire, the Cronbach $\alpha$ coefficient was used by participating the parents of 25 preterm infants. The obtained Cronbach $\alpha$ coefficient ranged from 0.76 to 0.80 .

In Ooman et al. (2011) study which used this questionnaire, the Cronbach $\alpha$ coefficient was reported between 0.81 and 0.96 . The sampling began after approval of the Ethics Committee of Tehran University of Medical Sciences (ethics code IR.TUMS.REC.1394.215) and receiving the introduction letter. Also, the directors of educational and treatment centers and associated departments in the research environment approved the study.

Furthermore, ethic principles were observed and the parents were assured about the confidentiality of their information. Obviously, the participants entered the study with full awareness of the aims of the study and by having filled out the consent forms. For the purpose of carrying out the study, the researcher attended the education-treatment centers and was personally in touch with parents of preterm infants who have been hospitalized for at least three days. The social support questionnaire was provided to each parent, and each of them individually filled out the questionnaire. Also, the demographic data of the infants and their parents were collected through the infant's files, and assistance of their parents. To compare the social support received by the father with the support received by the mother, data were analyzed using the Independent t test through SPSS version 20.

\section{Results}

Table 1 presents the demographic data of parents of preterm study infants in the three mentioned hospitals. The study did not have any sample attrition and 130 fathers of preterm infants and 130 mothers of the same infants participated in the study. Most childbirths were first time deliveries and also most of them were the result of cesarean section. The economic status of majority of the families was middle income.

Results indicated that the mean general support received by the fathers was 3.21 with standard deviation of 0.56 , and for mothers the mean received general support was 3.52 with standard deviation of 0.65 . These results showed that mothers received more supports in all three aspects of social support; affirmative, tangible, and emotional. Also, the highest level of social support received by fathers with mean(SD) score of 3.29(0.57) and mothers with mean(SD) score of 3.65(0.69) was emotional support and the least amount of support received by the fathers with mean(SD) score of 3.16(0.65) and mothers with mean(SD) score of $3.32(0.80)$ was the tangible support.

\section{Discussion}

Considering the family-centered care in the health administration of preterm infants and the quality of nursing cares, the present study was conducted to compare views of the fathers and mothers of preterm infants hospitalized at NICU regarding the social support they received. The measuring tool was the 21-item social support questionnaire, the results of which are discussed as follows. 
Table 1. Demographic data of parents of preterm study infants.

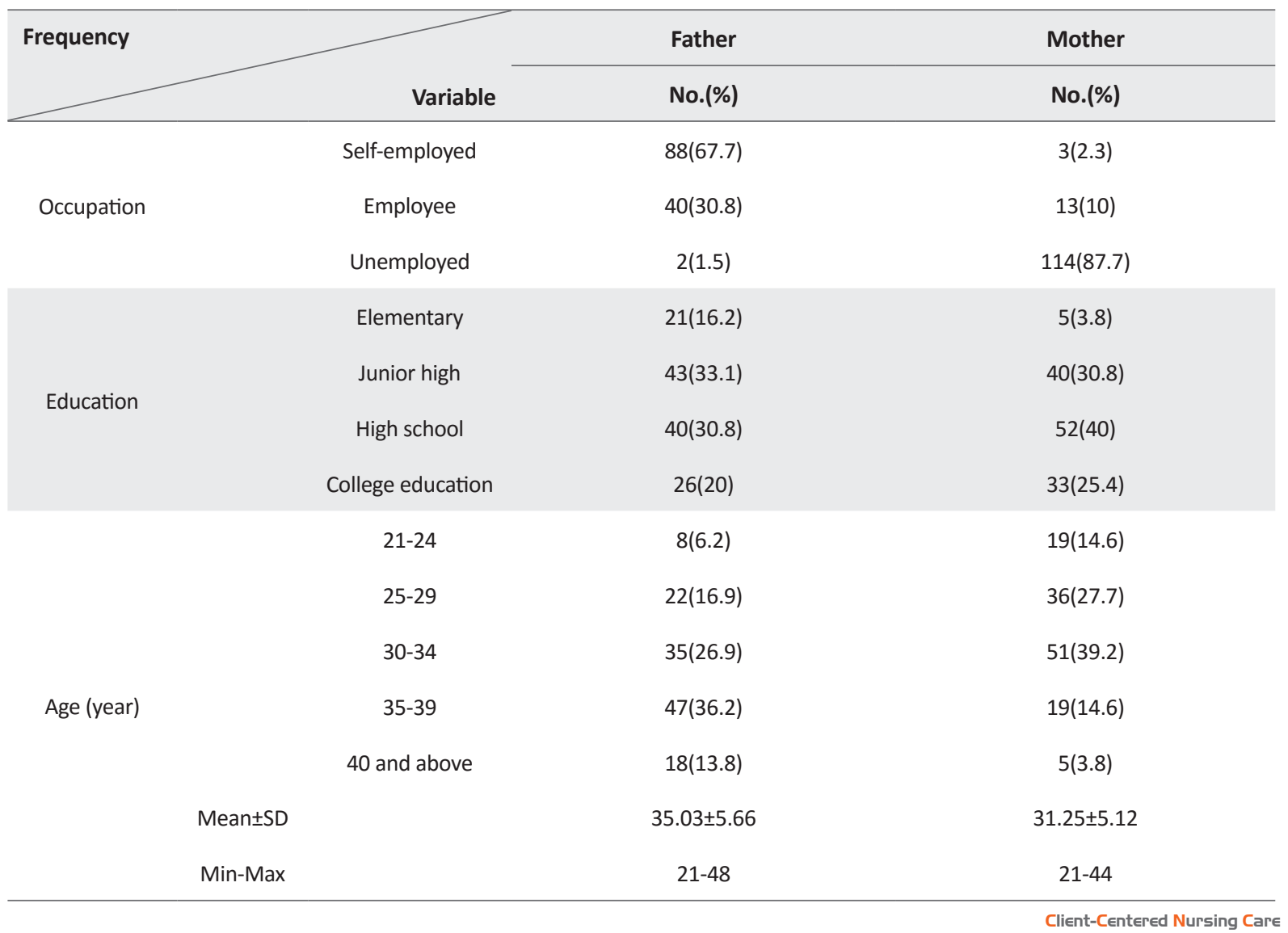

Table 2. Comparing the social support score and its aspects, between the fathers and mothers of preterm infants.

\begin{tabular}{|c|c|c|c|c|c|c|}
\hline \multirow{2}{*}{ Vupport subscale } & Father & Mother & Total & \multirow{2}{*}{ P-value } & \multirow{2}{*}{ df } & \multirow{2}{*}{$T$} \\
\hline & Mean $\pm S D$ & Mean \pm SD & Mean $\pm S D$ & & & \\
\hline Affirmative support & $3.17 \pm 0.69$ & $3.55 \pm 0.76$ & $3.36 \pm 0.67$ & \multirow{4}{*}{$\begin{array}{l}0.001 \\
\text { Paired t-test, } \\
\text { significant }\end{array}$} & \multirow{4}{*}{129} & \multirow{4}{*}{8.33} \\
\hline Tangible support & $3.16 \pm 0.65$ & $3.32 \pm 0.80$ & $3.24 \pm 0.61$ & & & \\
\hline Emotional support & $3.29 \pm 0.57$ & $3.65 \pm 0.69$ & $3.47 \pm 0.58$ & & & \\
\hline General support & $3.21 \pm 0.56$ & $0.65 \pm 3.52$ & $3.37 \pm 0.57$ & & & \\
\hline
\end{tabular}

Client-Centered Nursing Care

Results of the present study showed that in all aspects of social support, most parents needed support from nurses. The highest amount of support received by fathers and also by mothers was emotional support, and the least amount of support provided to the parents was the tangible support. Also, fathers as compared with mothers, received less support in any of the social support aspects (emotional, affirmative, tangible). Furthermore, the difference of social support received by fathers as compared with mothers, was statistically significant $(\mathrm{r}=0.766)$ (Table 2).
The statistical analysis showed that the parents received less tangible support and more emotional support. The Oomen et al. (2011) study showed that the social support parents received during the critical period of their infant hospitalization at NICU, is of utmost importance and nurses should be encouraged to acquire communication skills and create opportunities to provide social support for parents. The tools of their study for social support are used in the present study, too. In that study (Oomen et al. 2011), the data collection for the questionnaire was carried out after the infant's discharge; however, in our 
study, after the passage of 3 days of infant's hospitalization, data collection for the social support questionnaire was performed (Oomen et al. 2011). Also, in that study, fathers wanted more support and communication with the nurses which was due to inadequate communication the nurses had with the fathers. Parents needed more tangible support while they had received more emotional support. These results are similar to our study findings.

In Valizadeh et al. study (2009), the results showed that there was significant difference between mothers and nurses with regard to support (in all aspects) and its importance. Generally, mothers gave lower scores than nurses to every support subscale. In other words, NICU nurses expressed more importance for support subscales, and compared with the mothers, awarded higher scores to the support provided. In this study, mothers wanted more support than what they had received (Valizadeh et al. 2012).

In Borim Nejad et al. study (2011) aimed at identifying stressful factors as seen by mothers of preterm infants hospitalized at NICU, findings showed that parents of preterm infants, especially mothers, experience high levels of stress and a feeling of hopelessness in the NICUs, and this trend impacts the interaction between the infant and parents and the physical and emotional growth of the infants. In such situation and considering this study, some measures are needed for providing support to parents during the critical period of their infants' hospitalization in the NICU.

Based on the study findings, it is recommended that after the birth of the preterm child, required preparations be provided to the parents, especially to the mothers when meeting their infants in the NICU to establish appropriate relation with their infants (Borimnejad et al. 2011).

The study by Griffin has referred to creating opportunities for parents to help them with the care of their infants and to increase the parents' self-confidence. Similarly, in the present study, it has been demonstrated that the parents need support and encouragement by nurses in order to care for their infants (Griffin et al. 2008).

In Hynan (2005) study it is indicated that after the birth of preterm infant, it is possible that parents may not be able to receive information properly and would need special support and care, therefore, support for parents of preterm infant is very important (Hynan 2005).

In Sloan, Rowe, and Jones (2008) study only 50\% of fathers were satisfied of the information they received from the NICU personnel (Sloan et al. 2008). In Lindberg, Axelsson, and Ohrlin study (2007), fathers stated that they needed training and obtaining the information about their infants, therefore, it was very useful that nurses were accessible (Lindberg et al. 2008).

Among the limitations of this study was the noise and distractions in the NICUs during conversing with parents and filling out the questionnaire. Although the researcher made considerable efforts to control those limitations, at times it was not possible to fully control them.

The present study showed that parents received more emotional support and less tangible support. Also, fathers indicated that they received less support than that mothers received, which is probably due to fewer interactions of nurses with the fathers (as compared with the mothers), and mothers' greater role in caring for their infants. For this reason, it seems necessary that nurses in the NICU create more opportunities to communicate with and support for the fathers of preterm infants. In addition to providing training to the mothers for infant care, fathers also should receive necessary trainings, sufficient information, and adequate consideration.

Planning to provide more tangible support such as guidance for establishing communication with the infant, practical training for care and feeding the infant, and also material support seem necessary. It is recommended that the ways to supply support regarding every aspect be studied by nurses and included in nursing services. Also, considering the importance of family-centered care, nurses who spend the bulk of their service hours in the NICU with the infants, should provide more support and consideration to establish better communication with the parents of preterm infant, so that a step, even though a small one, be taken to improve family-centered care and health of the infant and to improve the quality of nursing services, too.

\section{Acknowledgments}

This study is part of the approved plan for the Master's thesis at Tehran University of Medical Sciences, with code No. 722. The researchers hereby express their appreciations for the financial support provided by Tehran University of Medical Sciences.

Also, we express our thanks and appreciation to all parents who participated in this study and to head nurses in the NICUs of Valiasr, Bahrami, and Arash treatment centers affiliated to Tehran University of Medical Sciences for their cooperation in carrying out this study in 2014

\section{Conflict of Interests}

The authors declared no conflict of interest. 


\section{Reference}

Arnold, L, Sawyer, A, Rabe, H, Abbott, J, Gyte, G, Duley, L et al . 2013, 'Parents' first moments with their very preterm babies: A qualitative study', BMJ, vol. 3, no. 4, p. e002487. doi: 10.1136/ bmjopen-2012-002487

Blencowe, H, Cousens, S, Oestergaard, MZ, Chou, D, Moller AB, Narwal, R et al. 2012, 'National, regional, and worldwide estimates of preterm birth rates in the year 2010 with time trends since 1990 for selected countries: a systematic analysis and implications', Lancet, vol. 379, no. 9832, pp. 2162-172. doi: 10.1016/s0140-6736(12)60820-4

Borimnejad, L, Seyyed Fatemi, N, Mehrnooh, N \& Haghani, H 2011, '[Maternal stressor agents with premature infants in neonatal intensive care units (Persian)]', Journal of Neonatal Intensive Care Nursing, vol. 4, no. 1, pp. 39-44.

Cleveland, LM 2008, 'Parenting in the neonatal intensive care unit', Journal of Obstetric, Gynecologic, \& Neonatal Nursing, vol. 37, no. 6, pp. 666-91. doi: 10.1111/j.1552-6909.2008.00288.x

Griffin, T, Wishba, C \& Kavanaugh, K 2008, 'Nursing interventions to reduce stress in parents of hospitalized preterm infants', Journal of Pediatric Nursing, vol. 13, no. 5, pp. 290-95. doi: 10.1016/s0882-5963(98)80014-6

Hynan, MT 2005, 'Supporting fathers during stressful times in the nursery: an evidence-based review', Newborn $\mathcal{E}$ Infant Nursing Reviews, vol. 5, no. 2, pp. 87-92. doi: 10.1053/j. nainr.2005.03.006

Ibrahimi, E, Mardani Hamoleh, M, Heidari, H, Mahboobi, M, 2011, '[Assessment of perceived social support among selected hospital personnel in Isfahan (Persian)]', Iranian Journal of Medical Ethics and History of Medicine, vol. 5, no. 1, pp. 82-88.

Johnson, AN 2008, 'Engaging fathers in the NICU: taking down the barriers to the baby', Journal of Prenatal $\mathcal{E}$ Neonatal Nursing, vol. 22, no. 4, pp. 302-6. doi: doi: 10.1097/01. JPN.0000341361.37822.34

Johnson, S, Ring, W, Anderson, P \& Marlow, N 2005, 'Randomized trial of parental support for families with very preterm children: outcome at 5 years', Archives of Disease in Childhood, vol. 90, no. 9, pp. 909-15. doi: 10.1136/adc.2004.057620

Lindberg, B, Axelsson, K \& Öhrling, K 2008, 'Adjusting to being a father to an infant born prematurely: experiences from Swedish fathers', Scandinavian Journal of Caring Sciences, vol. 22, no. 1, pp. 79-85.

Oommen, H, Rantanen, A, Kaunonen, M, Tarkka, MT \& Salonen, AH 2011, 'Social support provided to Finnish mothers and fathers by nursing professionals in the postnatal ward', Midwifery, vol. 27, no. 5, pp. 754-61. doi: 10.1016/j. midw.2010.06.017

Schappin, R, Wijnroks, L, Venema, MMU \& Jongmans, MJ 2013 'Rethinking stress in parents of preterm infants: a meta-analysis', PLoS One, vol. 8, no. 2, p. e54992.

Sikorova, L, Kucova, J 2012, 'The needs of mothers to newborns hospitalized in intensive care units', Biomedical Papers, vol. 156, no. 4, pp. 330-36. doi: 10.5507/bp.2011.046

Sloan, K, Rowe, J \& Jones, L 2008, 'Stress and coping in fathers following the birth of a preterm infant', Journal of Neonatal Nursing, vol. 14, no. 4, pp. 108-15. doi: 10.1016/j.jnn.2007.12.009
Valizadeh, ZV, Akbarbegloo, M \& Sayadi L 2012, 'Importance and availability of nursing support for mothers in NICU: A comparison of opinions of Iranian mothers and nurses', Iranian Journal of Pediatrics, vol. 22, no. 2, pp. 191-96. 